\title{
Differential Expression of Prolactin Releasing Peptide and Tyrosine Hydroxylase in Fibers Derived from the Same Neurons in the Rat
}

\author{
Kenshi Kakihara ${ }^{1, *}$, Norio Iijima ${ }^{2, *}$, Yoshihisa Naruse ${ }^{2}$, Yoshifumi Tanaka ${ }^{1}$, \\ Yasuhiko Ibata ${ }^{2,3}$ and Masaki Tanaka ${ }^{2}$ \\ ${ }^{1}$ Department of Anesthesiology, Kyoto Prefectural University of Medicine, Kawaramachi-Hirokoji, Kyoto 602-0841 and \\ ${ }^{2}$ Department of Anatomy \& Neurobiology, Kyoto Prefectural University of Medicine, Kawaramachi-Hirokoji, Kyoto 602-0841 \\ ${ }^{3}$ Present affiliation: Office of the President, Kyoto Prefectural University of Medicine
}

Received November 21, 2002; accepted January 7, 2003

\begin{abstract}
We found differential expression of two neuronal markers in nerve fibers that came from the same neuronal somata. Prolactin releasing peptide (PrRP) and tyrosine hydroxylase (TH), a catecholamine synthesis rate-limiting enzyme, were differentially distributed in distinct fibers derived from the same neuronal cell body located in the rat medulla oblongata. All PrRP-immunoreactive neurons were catecholaminergic at the cell body level in the nucleus tractus solitarius (NTS) and in the ventral and lateral reticular nuclei (VLRN). However, in the terminal areas such as the bed nucleus of the stria terminalis (BNST) and paraventricular nucleus (PVN), where
\end{abstract}

fibers are projected from both PrRP and TH positive neurons in the NTS and VLRN, dense PrRP immunoreactive fibers were observed that did not contain TH. In the medulla oblongata, proximal fibers near a neuronal cell body that contained both TH and PrRP were diverged into mainly PrRP-positive or mainly TH-positive fibers. These results suggest that TH and PrRP are differentially expressed between fibers from the same cell body. The fibers containing predominantly PrRP have been detected at birth, so the machinery to transport PrRP and TH separately may be established at an embryonic stage.

Key words: Prolactin-releasing peptide, Tyrosine hydroxylase, Immunocytochemistry, Neuronal projection, Rat

\section{Introduction}

Prolactin-releasing peptide (PrRP) was originally isolated from bovine hypothalamus on the basis of its ability to stimulate an orphan G protein-coupled receptor, hGR3 [5]. PrRP plays important roles in the hypothalamic neuroendocrine system, and influences the autonomic nervous system and several behaviors [9, 13, 16, 24, 28]. PrRP containing neurons are located exclusively in the nucleus tractus solitarius (NTS), in the ventral and lateral reticular nuclei (VLRN) in the medulla oblongata, and in the dorsomedial hypothalamic nucleus $[3,8,12]$.

In the NTS and VLRN, PrRP are co-expressed in the

\footnotetext{
* These authors contributed equally to this work.
}

Correspondence to: Masaki Tanaka, M.D., Ph.D., Department of Anatomy \& Neurobiology, Kyoto Prefectural University of Medicine, Kawaramachi-Hirokoji, Kyoto 602-0841, Japan. same neuronal cell body with the catecholamine-synthesizing enzyme, tyrosine hydroxylase $(\mathrm{TH})$, by immunocytochemistry $[3,8]$. The PrRP-immunoreactive fibers are broadly distributed in the forebrain; in the bed nucleus of the stria terminalis (BNST), the paraventricular nucleus (PVN), the periventricular nucleus (PerVN), the supraoptic nucleus (SON), the corticomedial amygdaloid nucleus (CAM) and the mediodordsal thalamic nucleus (ThMDN) $[6,8,12]$. Among them, fibers are particularly densely distributed in the BNST region. In the BNST, PrRP-immunoreactive axon terminal made synapses and distribution of the PrRP receptor (UHR-1) have been observed [6, 23]. However, the origin of PrRP fibers in the forebrain has yet to be clarified. In the present study, we investigated the distribution of PrRP and TH in the fibers of the forebrain, and sought the origin of the PrRP fibers in the BNST by using a retrograde tracer.

Unexpectedly we found almost distinct expression of PrRP- and TH-immunoreactive fibers in the BNST and the 
PVN while BNST and PVN-projecting neurons contained both PrRP and TH fibers at the neuronal cell body in the medulla oblongata.

\section{Materials and Methods}

\section{Animals}

Male Wistar rats weighing about $250 \mathrm{~g}$ were purchased from SHIMIZU Laboratory Supplies Co. Ltd. (Kyoto, Japan). Newborn rats at birth were obtained by breeding. Food and water were provided ad libitum, and the animals were kept under controlled lighting ( $12 \mathrm{hr}$ light-12 hr dark) and temperature $\left(22^{\circ} \mathrm{C}\right)$ until used for experiments. All experiments in this study were carried out following the National Institutes of Health Guidelines for the Care and Use of Laboratory Animals. The experiments were approved by the Committee of Animal Research in Kyoto Prefectural University of Medicine.

\section{PrRP and TH immunocytochemistry}

Under deep anesthesia with sodium pentobarbital, adult male and new born rats were perfused with $200 \mathrm{ml}$ and $20 \mathrm{ml}$, respectively, with $0.1 \mathrm{M}$ phosphate buffer containing $4 \%$ formaldehyde. Brains were removed and postfixed overnight [15]. Serial frozen sections $(30 \mu \mathrm{m})$ were cut from the forebrain and medulla oblongata. Sections were incubated with monoclonal antibody P2L-1C $(10 \mu \mathrm{g} / \mathrm{ml})$ and P2L-1T $(10 \mu \mathrm{g} / \mathrm{ml}$, gifts from Dr. Hinuma) in PBS containing $0.5 \%$ triton $\mathrm{X}-100$ at $4{ }^{\circ} \mathrm{C}$ for 2 days. P2L-1C reacts with PrRP31 and PrRP20, and P2L-1T recognizes PrRP-prepropeptide $[12,16]$. In order to clearly detect PrRP in the cell body and fibers on the same section, sections were incubated with both P2L-1C (10 $\mu \mathrm{g} / \mathrm{ml})$ and P2L-1T $(10 \mu \mathrm{g} / \mathrm{ml})$ for 2 days. As a second antibody, Cy3 (1,1-bis(epsilon-carboxypentyl)3,3,3',3'-tetramethylindocarbocyanine-5,5'-disulfonate)-conjugated anti-mouse IgG antibody (dilution; 1:1000, Chemicon International Inc., Temecula, CA) was used for the detection of PrRP. For double labeling immunocytochemistry of PrRP and TH, sections were incubated with antiTH antiserum (dilution; 1:5000, a gift from Prof. Nagatsu), P2L-1T and P2L-1C. As second antibodies, Cy3-conjugated anti-mouse IgG antibody and fluorescein isothiocyanate (FITC)-conjugated anti-rabbit $\mathrm{IgG}$ antibody (dilution; 1:1000, Vector laboratories, Inc., Burlingame, CA) were used for the detection of PrRP and TH, respectively. Sections were mounted onto glass slides and examined by fluorescent microscopy (Nikon, Tokyo, Japan) or by confocal laser microscopy (Olympus Fluoview, Olympus, Tokyo, Japan).

The specificity of these antibodies was described previously $[4,7,12,20]$.

\section{The retrograde tracing in PrRP fibers to examine the origin of the PrRP nerve terminals}

The retrograde tracing experiments were performed as previously reported [25]. Male adult rats were anesthetized by intraperitoneal administration of $50 \mathrm{mg} / \mathrm{kg}$ sodium pento- barbital, and then set in a rat brain stereotaxic apparatus (Neuroscience, Japan). A Hamilton microsyringe ( $1 \mu \mathrm{l})$ was used to inject a retrograde tracer, cholera toxin B subunit (CTb). A fine glass pipette made with a micropipette puller (Takahishi Co., Japan) was attached to the tip of the syringe. A small hole was drilled in the parietal skull, and a glass pipette inserted into the right BNST (anterior-posterior: $+9.20 \mathrm{~mm}$ from the interaural line, lateral: $1.00 \mathrm{~mm}$ from the midline, height: $+2.80 \mathrm{~mm}$ from the interaural line) according to the atlas of Paxinos [20]. 0.1 $\mu$ l of CTb (50 $\mathrm{mg} / 50 \mu \mathrm{l}$ dissolved in distilled water, Sigma, USA) was slowly pressure-injected. After 3 days, rats were perfused with the same fixative as described above. Sections from the medulla oblongata and caudal hypothalamus were incubated with PL2-1T and anti-CTb antiserum (dilution 1:5000, List Biochemical Laboratories) for 2 days. As second antibodies, Cy3-conjugated anti-mouse IgG anti-
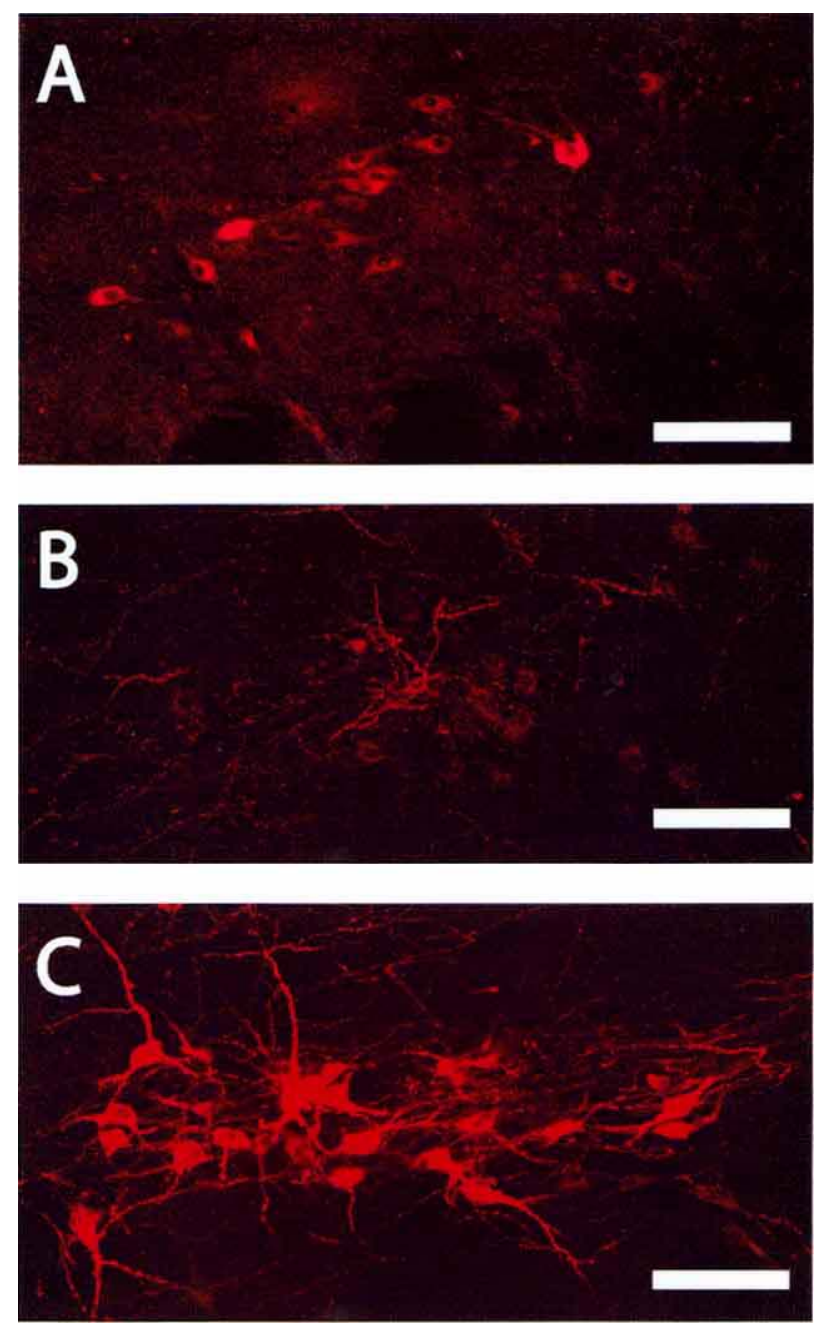

Fig. 1. Immunocytochemistry with PL2-1T (A) and PL2-1C (B) in the NTS. A: PL2-1T immunoreactivity is localized in cell bodies. B: PL2-1C immunoreactivity is detected in the nerve fibers. C: The combination of PL2-1T and PL2-1C recognizes both PrRP-positive cell bodies and fibers. Bar $=100 \mu \mathrm{m}$. 
body for the detection of PrRP and FITC-conjugated antigoat IgG antibody (dilution; 1:1000, Vector laboratories, Inc., Burlingame, CA) were used for the detection of $\mathrm{CTb}$ were used.

\section{Cell counting}

All sections from the medulla oblongata and the hypothalamus where PrRP neurons were detected were taken for cell counting. All PrRP-immunoreactive neuronal cell bodies were counted by examining the co-expression with TH (number of animals, 6). Three animals into which the tracer had been properly injected in the BNST were used for the retrograde tracing experiment. All PrRP-immunoreactive neuronal cell bodies in the hypothalamus and medulla oblongata were counted by examining the co-labeling with $\mathrm{CTb}$.

\section{Results}

\section{Immunocytochemical characterization of PrRP neurons}

Fluorescent PL2-1T immunoreactivity was predominantly detected in cell bodies and slightly in the fibers (Fig. 1A). However, PL2-1C immunoreactivity was observed mainly in the fibers (Fig. 1B). The immunocytochemistry with a combination of these antibodies revealed that strong signals were observed in both cell bodies and fibers on the same section (Fig. 1C). Therefore, we investigated the distribution of PrRP using this combination of PL2-1T and PL21C. There were only three regions where PrRP positive neuronal cell bodies were detected; in the NTS and VLRN in medulla oblongata, and in the dorsomedial nucleus in the hypothalamus. PrRP-positive neurons were predominantly distributed in the medulla oblongata and less than $10 \%$ of the total PrRP positive cells were observed sparsely in the hypothalamus. In the NTS and VLRN, PrRP-immuno-
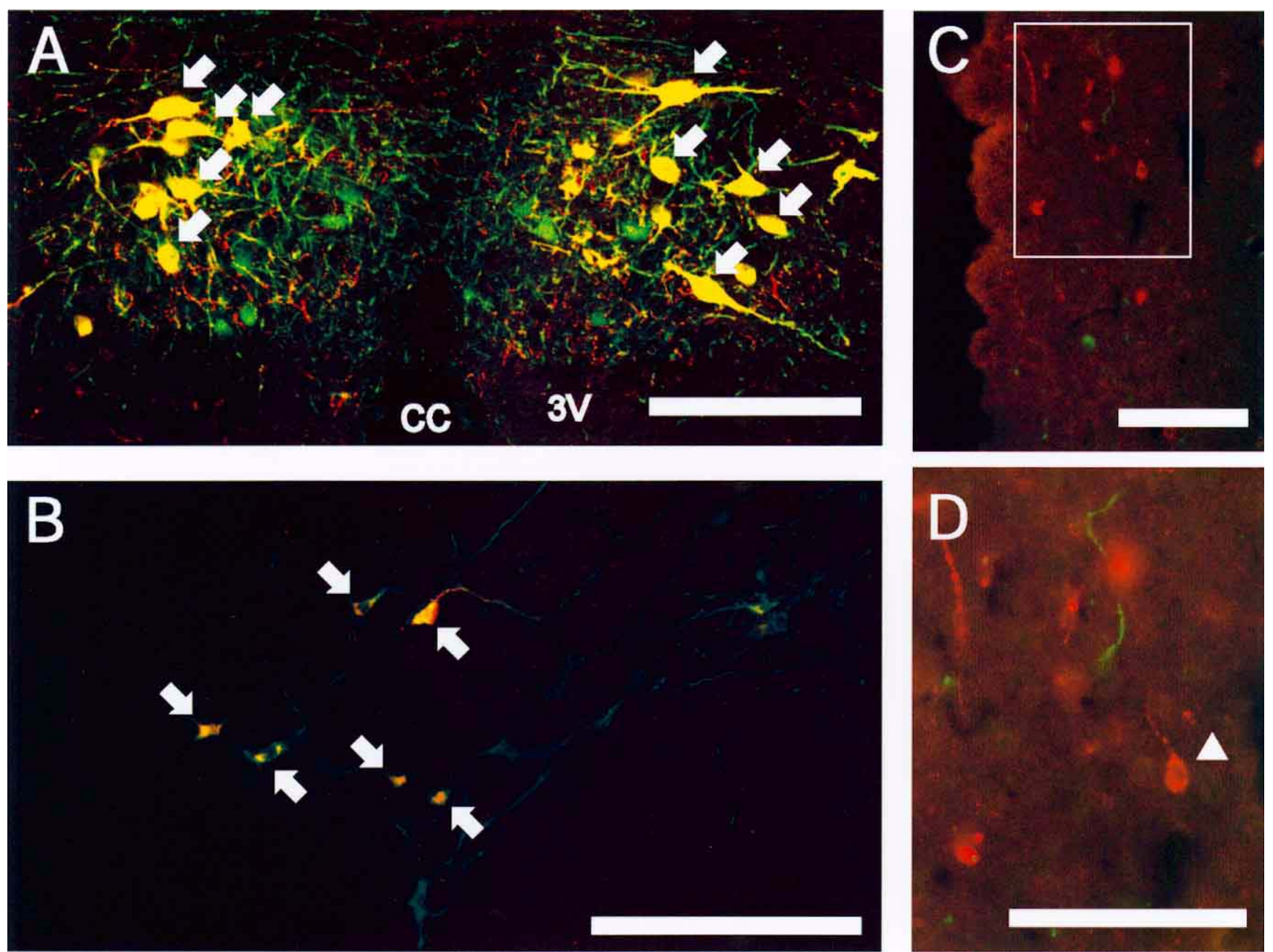

Fig. 2. Double labeling immunocytochemistry with PrRP and tyrosine hydroxylase in the NTS (A), VLRN (B) and hypothalamic dorsomedial nucleus (C, D). Red Cy3 staining shows PrRP and green FITC staining shows TH. All PrRP immunoreactivity is co-localized with TH in the cell bodies and turns yellow in the NTS (A) and VLRN (B). In the NTS, there are fibers that show only red PrRP immunoreactivity. Arrows indicate cell bodies in which PrRP and TH are co-localized. On the other hand, in the hypothalamus, PrRP immunoreactive cell bodies do not contain any TH (arrow head) (C, D). The square in $\mathbf{C}$ is magnified in the photograph $\mathbf{D}$. Bar=100 $\mu \mathrm{m}$. cc, central canal; 3V, third ventricle. 
Table 1. Co-localization of PrRP and TH in the cell bodies in the NTS and the VLRN

\begin{tabular}{lcccc}
\hline & Animal & $\begin{array}{c}\text { Number of } \\
\text { PrRP cells }\end{array}$ & $\begin{array}{c}\text { Number of } \\
\text { TH cells }\end{array}$ & $\begin{array}{c}\text { Number of } \\
\text { PrRP+TH cells }\end{array}$ \\
\hline NTS & 1 & 338 & 519 & 338 \\
& 2 & 345 & 536 & 345 \\
& 3 & 340 & 551 & 340 \\
& 4 & 298 & 513 & 298 \\
& 5 & 311 & 521 & 311 \\
& 6 & 292 & 537 & 292 \\
\hline VLRN & & & & \\
& 1 & 62 & & 62 \\
& 2 & 75 & & 58 \\
& 3 & 58 & & 67 \\
& 4 & 67 & & 50 \\
\hline
\end{tabular}

reactive neurons always contained $\mathrm{TH}$ at the cell body (Fig. 2A, B). The number of PrRP-immunoreactive cell bodies was coincident with the number of cell bodies that were double-labeled with PrRP and TH. Conversely, sixty per cent of the TH-immunoreactive neurons in the NTS contained PrRP (Table 1). However, in the hypothalamus, scattered PrRP-immunoreactive neurons did not co-express TH (Fig. 2C, D).

\section{Immunocytochemical characterization of PrRP fibers}

In the BNST, PVN, ThMDN, SON and CAN, many red PrRP-immunoreactive fibers were observed intermingled with TH-immunoreactive fibers, which looked green. In the BNST, below the anterior commissure where PrRP fibers were the most densely distributed, they were intermingled with TH fibers (Fig. 3A). Under higher magnified observation, we could almost discriminate red fibers which contained PrRP alone and green fibers which contained $\mathrm{TH}$ alone (Fig. 3B). In the portion around the BNST between the lateral ventricle and the anterior commissure, green $\mathrm{TH}$ fibers were dominantly observed (Fig. 3A). In the PVN, both PrRP and TH fibers were observed. PrRP fibers were observed mainly in the lateral portion of PVN (Fig. 3C), and relatively dense $\mathrm{TH}$ fibers were located in the periventricular area. These PrRP fibers in the PVN looked red with scanty TH immunoreactivity (Fig. 3D). In the thalamus, PrRP fibers were restricted within the ThMDN. TH fibers were observed from the paraventricular thalamic nucleus to the ThMDN. In the ThMDN, most of the PrRP-immunoreactive fibers also contained TH, which looked yellow (Fig. 3E, F). In the SON and CAN, some PrRP fibers contained TH but others did not express TH (data not shown).

In respect to pure PrRP fibers not containing $\mathrm{TH}$, we excluded the possibility that anti-PrRP antibody could inhibit the anti-TH antibody recognizing $\mathrm{TH}$ in the same fiber in an unknown manner. Initially we applied only anti$\mathrm{TH}$ antiserum to the sections. After washing with PBS, the sections were incubated with anti-PrRP antibody (P2L-1C). Immunostaining by this procedure coincided with the results of applying PrRP and TH antibodies together. We also tried another TH antibody commercially available (Chemicon Inc.) and obtained the same results that TH and PrRP were differentially distributed in the BNST (data not shown).

\section{Retrograde tracing of PrRP fibers}

We retrogradely traced PrRP fibers in the BNST to exclude the possibility that all PrRP-immunoreactive fibers detected in the BNST could be derived from the PrRPpositive hypothalamic neurons that did not express $\mathrm{TH}$. After injection of $\mathrm{CTb}$ into the ventral right BNST (Fig. 4A), CTb was detected in the PrRP-immunoreactive cell bodies in the NTS, VLRN and hypothalamus (Fig. 4B-E). Quantitative analysis showed that about 50\% (145/294.7) of the PrRP-positive neurons had CTb in the ipsilateral NTS and that about $70 \%(82 / 117.3)$ of the PrRP-positive neuron had $\mathrm{CTb}$ in the ipsilateral VLRN (Fig. 4B, C). In this experiment, 95\% $(259 / 274 ; 145+10+82+22 / 8.7+6.3$ $+145+10+82+22)$ of neurons containing CTb and PrRP were located in the medulla oblongata (Table 2). We also detected $\mathrm{CTb}$ immunoreactivity in some PrRP-positive neurons in the hypothalamus (Fig. 4D, E).

\section{Segregated distribution of PrRP and TH in fibers of the NTS}

In the NTS, where both PrRP and TH containing cell bodies (yellow) exist, only red or only green colored fibers were detected (Figs. 2A, 5A). Highly magnified microscopy revealed differential immunostaining against PrRP and $\mathrm{TH}$ in the fibers of the same neuron (Fig. 5B). At the proximal region, fibers were initially yellow (both PrRP-and THpositive). Then, one fiber turned only red (PrRP-positive without TH) and the other turned green (TH-positive without PrRP).

\section{Distribution of PrRP and TH in fibers of the neonatal brain}

In the brain at birth, when axonal projections of PrRP immunoreactive fibers were under construction, fibers were already only red or only green around the BNST (Fig. 6A, B) and PVN (Fig. 6C, D). PrRP positive fibers did not coexpress $\mathrm{TH}$ in these regions.

\section{Discussion}

PrRP-producing cells are located in the NTS, VLRN in the medulla oblongata and in the dorsomedial nucleus in the hypothalamus [9, 18]. Quantitative in situ hybridization analysis showed that approximately $70 \%$ of the total PrRP mRNA in the brain, and that more than $20 \%$ of that quantity was located within the NTS and VLRN, respectively [10]. On the other hand, enzyme immunoassay and bioassay revealed that the highest levels of PrRP were detected in the hypothalamus and that moderate levels of PrRP were detected in the midbrain $[4,16]$. These results suggest that most PrRP is synthesized in the medulla oblongata and 

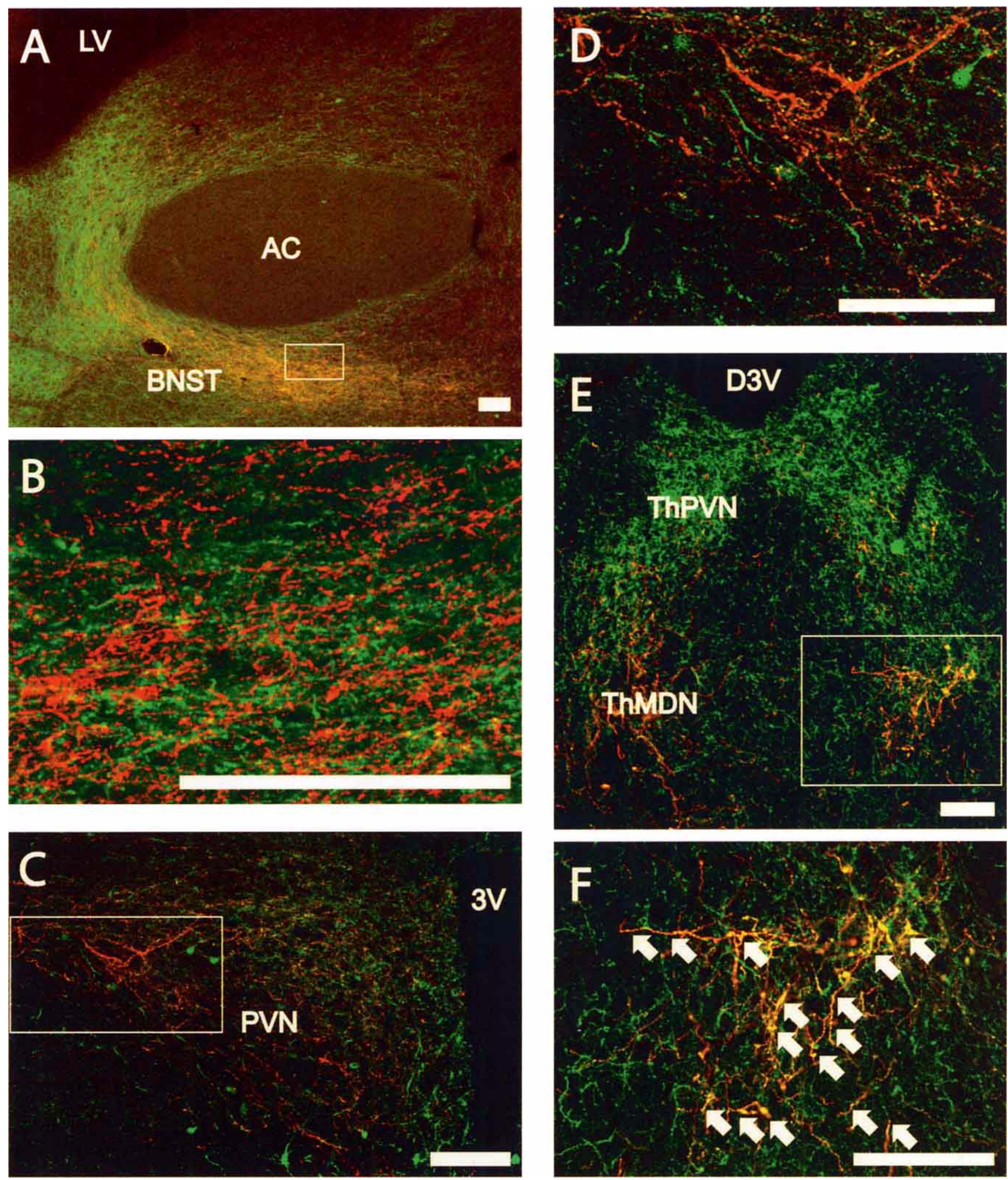

Fig. 3. Distribution of PrRP and TH in the nerve fibers. In the BNST below the anterior commissure, TH- and PrRP-immunoreactive fibers are intermingled (A). In higher magnification, PrRP-immunoreactive fibers exhibit only red color and do not express green TH (B). In the PVN, PrRP fibers are denser in the lateral portion. TH immunoreactivity is strong in the medial portion (C). Red PrRP fibers do not contain green TH at all (D). In the ThMDN, most of PrRP fibers appear yellow (E, F). Arrows indicate the fibers containing both PrRP and TH. The squares in A, $\mathbf{C}$ and $\mathbf{E}$ are magnified as the photographs $\mathbf{B}, \mathbf{D}$ and $\mathbf{F}$ respectively. The sections are sagittal in $\mathbf{A}, \mathbf{B}$ and coronal in $\mathbf{C}, \mathbf{D}, \mathbf{E}, \mathbf{F}$. AC, anterior commissure; BNST, bed nucleus of stria terminalis; D3V, dorsal third ventricle; LV, lateral ventricle; PVN, paraventricular hypothalamic nucleus; ThMDN, mediodorsal thalamic nucleus; ThPVN, paraventricular thalamic nucleus; $3 \mathrm{~V}$, third ventricle. Bar $=100 \mu \mathrm{m}$. 

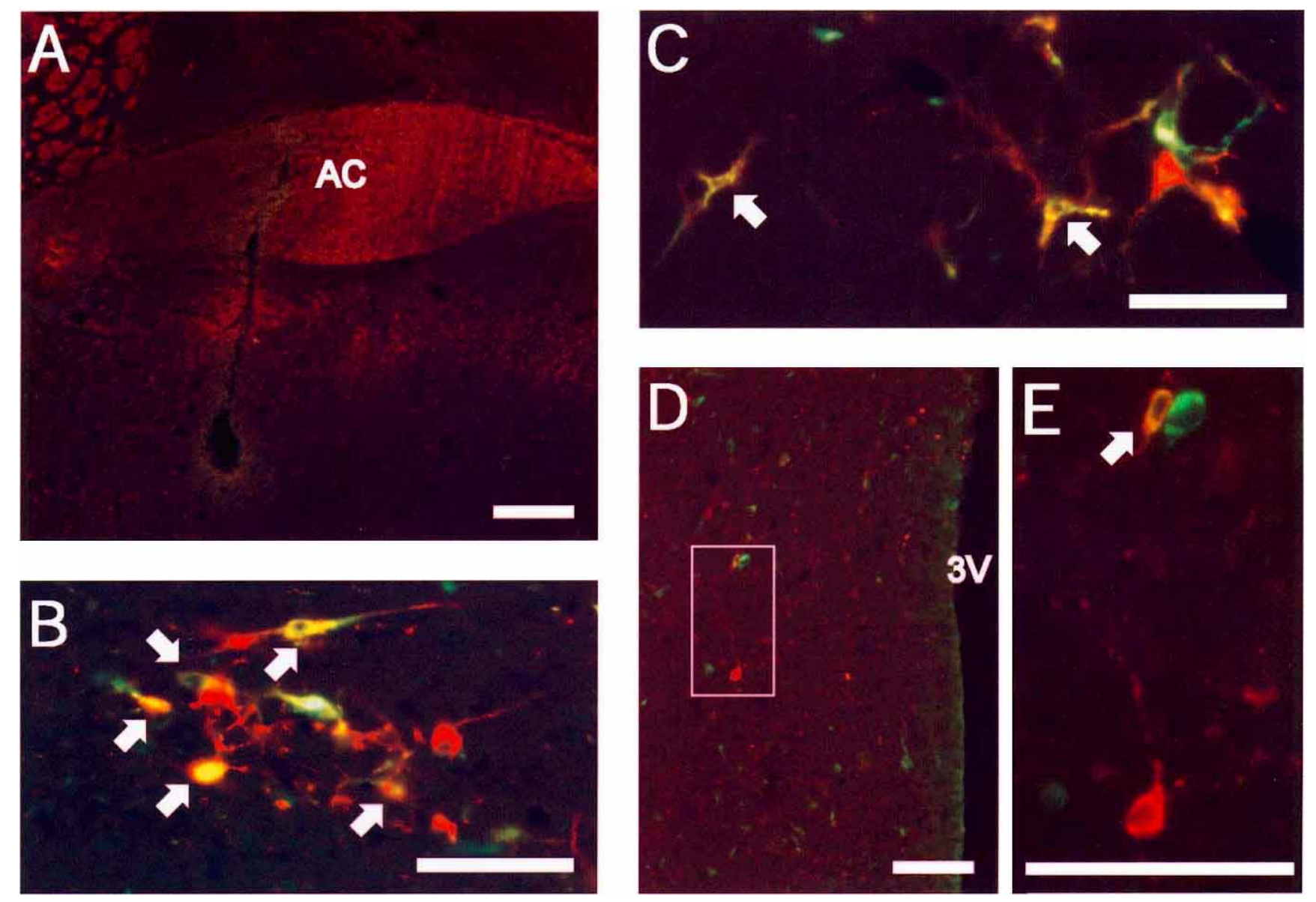

Fig. 4. Detection of the retrograde tracer CTb injected into the BNST. A: injection site of CTb in the right BNST. CTb labeled cell bodies are detected in the NTS (B), VLRN (C) and the hypothalamus (D, E). Co-localization with PrRP was examined. Green and red fluorescence show $\mathrm{CTb}$ and PrRP respectively. Arrowed neurons that show both CTb and PrRP are observed in the NTS (B) and VLRN (C). There are a few colocalized neurons in the hypothalamus (D, E). The square in the photograph $\mathbf{D}$ is magnified in the photograph as $\mathbf{E}$. AC, anterior commissure; $3 \mathrm{~V}$, third ventricle. Bar $=100 \mu \mathrm{m}$.

Table 2. Number of CTb-positive neurons in PrRP neurons

\begin{tabular}{|c|c|c|c|c|c|c|}
\hline \multirow{2}{*}{ Animal } & \multicolumn{2}{|c|}{ Hypothalamus } & \multicolumn{2}{|c|}{ NTS } & \multicolumn{2}{|c|}{ VLRN } \\
\hline & ipsi & contra & ipsi & contra & ipsi & contra \\
\hline 1 & $8 / 54$ & $5 / 44$ & $138 / 298$ & $9 / 258$ & $71 / 110$ & $18 / 92$ \\
\hline 2 & $8 / 50$ & $6 / 48$ & $125 / 271$ & $11 / 275$ & $77 / 100$ & $16 / 110$ \\
\hline 3 & $10 / 61$ & $8 / 59$ & $172 / 315$ & $10 / 281$ & $98 / 142$ & $32 / 107$ \\
\hline mean & $8.7 / 55$ & $6.3 / 50.3$ & $145 / 294.7$ & $10 / 271.3$ & $82 / 117.3$ & $22 / 103$ \\
\hline
\end{tabular}

transported to the hypothalamus and other rostral parts of the brain $[6,9,12]$. In this study, we showed that all PrRP neurons in the medulla oblongata have a catecholaminergic neuronal profile. Therefore, it was anticipated that PrRP positive fibers coming from the medulla oblongata in the forebrain would exhibit a marker of the catecholaminergic neuron, TH. However, PrRP-immunoreactive fibers in the BNST and PVN did not co-express TH, while PrRP-immunoreactive fibers in the ThMDN did. TH has been used as a marker of catecholaminergic neuronal somata and fibers for various morphological studies of the catecholaminergic system in the brain. The anti-TH antibody used in the present study has been established for immunocytochemistry against TH [7, 19, 25]. Even though there was a possibility that TH was contained in PrRP positive fibers in the BNST and the PVN under detection level, the difference in expression of PrRP and TH apparently existed. From this result, PrRP-immunoreactive fibers could be divided into two categories reflecting the content of TH on each fiber. One is a fiber like PrRP fiber in the BNST and PVN, which 

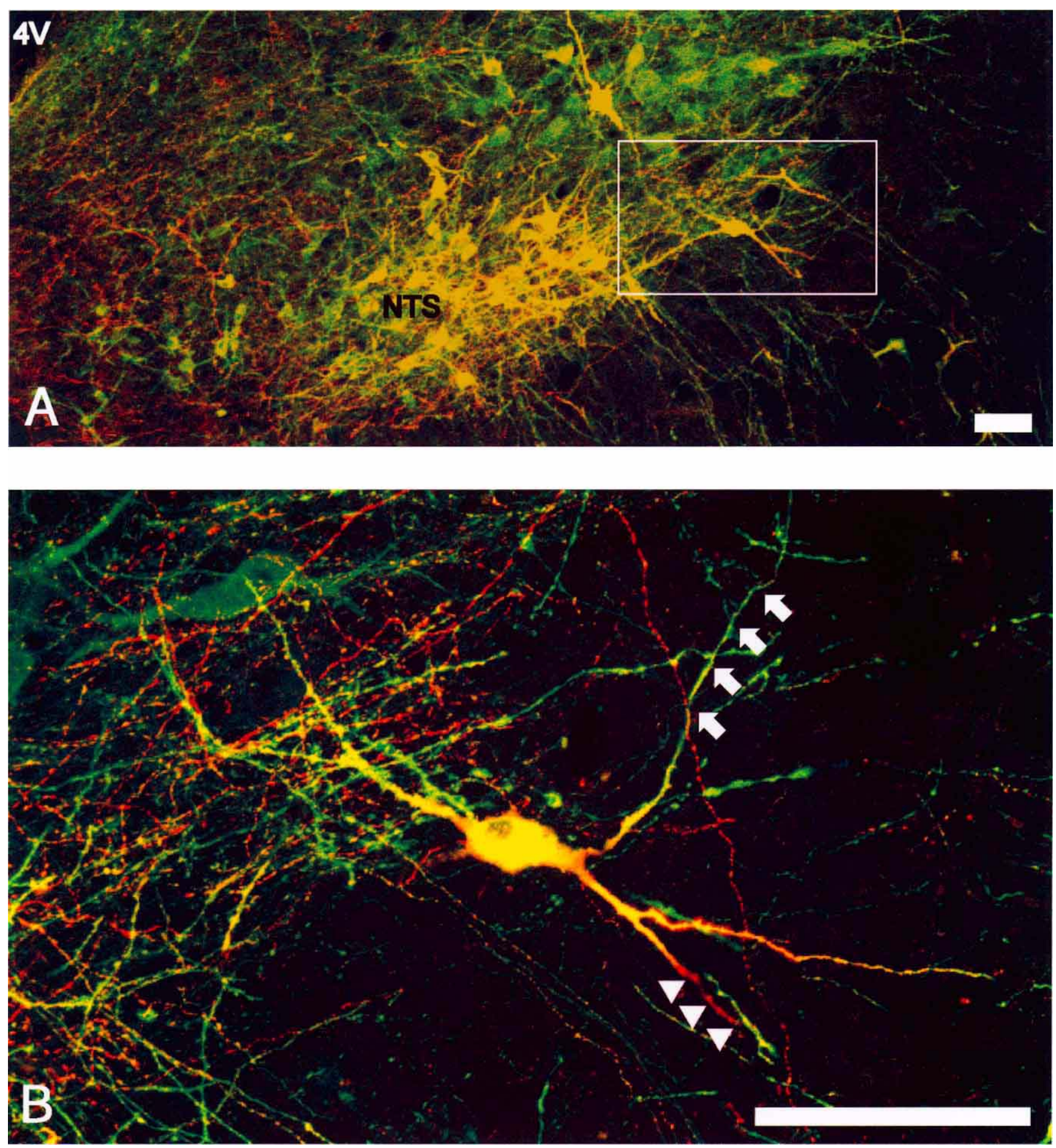

Fig. 5. Differential distribution of PrRP and TH in the NTS. All PrRP immunoreactive neuronal cell bodies co-express TH and look yellow (A). Photograph $\mathbf{B}$ is a higher magnified picture of a square in A. The fibers from one PrRP-positive neuron diverge into two types of branches at the proximal region. Arrows indicate that the fibers diminish PrRP but remain TH. Arrowheads indicate the fiber showing only PrRP without TH. Sections are horizontal. NTS, nucleus tracts solitarius; 4V, fourth ventricle. Bar $=100 \mu \mathrm{m}$.

do not or co-express $\mathrm{TH}$ at a very low level. The other is a type of fiber detected in the thalamus, which also coexpresses TH simultaneously. In the SON and the CAN, both types of fibers exist.

In this study, experiments using a retrograde tracer,
$\mathrm{CTb}$, and immunocytochemistry against PrRP showed that PrRP neurons in the medulla oblongata projected their nerve fibers to the BNST far more than those in the hypothalamus did. Morales et al. reported that numerous PrRP cells in the medulla oblongata were retrogradely labeled following 

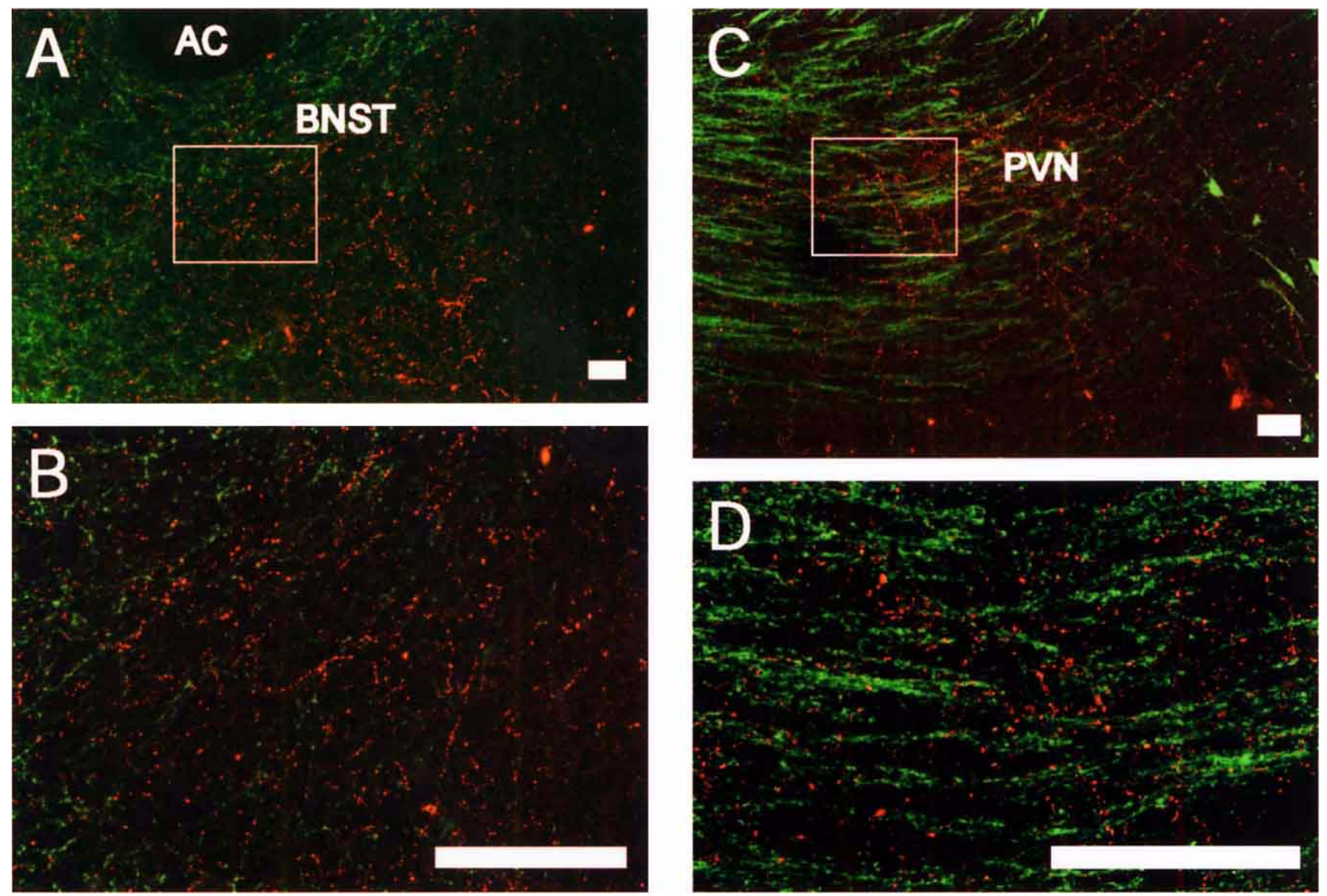

Fig. 6. Immunocytochemistry against PrRP and TH in the nerve fibers of the neonatal brain. PrRP-immunoreactive fibers are distributed in the BNST (A, B) and the PVN $(\mathbf{C}, \mathbf{D})$ at the day of birth. The squares in $\mathbf{A}$ and $\mathbf{C}$ are magnified in the photograph as $\mathbf{B}$ and $\mathbf{D}$, respectively. These red PrRP fibers do not contain any green TH. AC, anterior commissure; LV, lateral ventricle; $3 \mathrm{~V}$, third ventricle. Bar=100 $\mu \mathrm{m}$.

tracer injections into the PVN [19]. These results seem to exclude the possibility that all PrRP-immunoreactive fibers located in the BNST and the PVN could be derived from PrRP neurons in the hypothalamus, in which PrRP neurons did not express TH. In the pons, most PrRP fibers, which were thought to be on the way to the forebrain from the NTS/VLRN region, did not express TH (data not shown). Therefore, TH may be diminished in the proximal region of most of PrRP-fibers from both PrRP- and TH-positive cell bodies in the medulla oblongata.

Then, we detected the differential distribution of PrRP and $\mathrm{TH}$ into distinct fibers derived from the same neuronal cell body in the NTS, as shown in Fig. 5. This result indicates that PrRP and TH may be mainly transported to the target region along separated nerve fibers. As there is a possibility that some fibers in Fig. 5 are neuronal dendrites, at least in some neurons, PrRP and TH are differentially distributed in the level at proximal processes. Considering that ascending PrRP fibers in the pons are observed without containing TH, it is probable that some PrRP neurons have different axonal fibers with neurotransmission systems. Some neuronal cytoskeletal proteins are already known to have segregated distribution. Microtubule-associated protein
2 (MAP2) is dominantly localized in somatodendrite [1], while tau is abundant in axons [2, 10, 22]. However a segregated distribution in axon branches has not been previously reported. The transporting system of PrRP and TH could be different in those distinctively distributed fibers. Some kinds of motor protein may play roles in the distinctive axonal transports of PrRP and TH.

Recently Maruyama et al. showed that PrRP was a stress mediator and modulated the hypothalamo-pituitaryadrenal axis co-operatively with noradrenaline [14]. They showed that PrRP and noradrenaline synergistically play roles in the secretion of ACTH. In this study, we observed that the fibers containing only PrRP were always intermingled with TH-immunoreactive fibers in the BNST and PVN. Even though PrRP and $\mathrm{TH}$ are transported along different fibers from the same cell body, PrRP and presumably synthesized noradrenaline may act co-operatively in the target region.

At the time of birth, PrRP immunoreactive cell bodies and PrRP mRNA were not detected in the hypothalamus and the distribution of PrRP fibers is not as developed as in adult rat [27]. However, at this stage, PrRP and TH were colocalized in the neuron of the medulla oblongata [27] and fibers 
with only PrRP immunoreactivity were detected around targeted areas in the brain. Distinct systems for the respective transportation of PrRP and TH could already be established in developing neurons in newborn rats.

In conclusion, we found that two neurotransmission systems diverged into distinct fibers derived from the same catecholaminergic neuron. It is expected that other kinds of neurons could also have the possibility of differential neurotransmission systems in their distinct neuronal fibers.

\section{Acknowledgments}

We are grateful to Emeritus Professor I. Nagatsu (Fujita Health University of Medicine, Aichi, Japan) for kindly providing the antisera to TH and grateful to Dr. S. Hinuma (Takeda Chemical Industries Ltd.) for P2L-1C and P2L-1T. This investigation was supported by grants from the Ministry of Education, Science and Culture, Japan.

\section{References}

1. Bernhardt, R. and Matus, A. (1984) Light and electron microscopic studies of the distribution of microtubule-associated protein 2 in rat brain: a difference between dendritic and axonal cytoskeletons. J. Comp. Neurol. 226; 203-221.

2. Binder, L. I., Frankfurter, A. and Rebhun, L. I. (1985) The distribution of tau in the mammalian central nervous system. J. Cell Biol. 101; 1371-1378.

3. Chen, C., Dun, S. L., Dun, N. J. and Chang, J. K. (1999) Prolactin-releasing peptide-immunoreactivity in A1 and A2 noradrenergic neurons of the rat medulla. Brain Res. 822; 276-279.

4. Fujii, R., Fukusumi, S., Hosoya, M., Kawamata, Y., Habata, Y., Hinuma, S., Sekiguchi, M., Kitada, C., Kurokawa, T., Nishimura, O., Onda, H., Sumino, Y. and Fujino, M. (1999) Tissue distribution of prolactin-releasing peptide (PrRP) and its receptor. Regul. Pept. 83; 1-10.

5. Hinuma, S., Habata, Y., Fujii, R., Kawamata, Y., Hosoya, M., Fukusumi, S., Kitada, C., Masuo, Y., Asano, T., Matsumoto, H., Sekiguchi, M., Kurokawa, T., Nishimura, O., Onda, H. and Fujino, M. (1998) A prolactin-releasing peptide in the brain. Nature 393; 272-276.

6. Ibata, Y., Iijima, N., Kataoka, Y., Kakihara, K., Tanaka, M., Hosoya, M. and Hinuma, S. (2000) Morphological survey of prolactin-releasing peptide and its receptor with special reference to their functional roles in the brain. Neurosci. Res. 38; 223-230.

7. Ichitani, Y., Okamura, H., Nakahara, D., Nagatsu, I. and Ibata, Y. (1994) Biochemical and immunocytochemical changes induced by intrastriatal 6-hydroxydopamine injection in the rat nigrostriatal dopamine neuron system: evidence for cell death in the substantia nigra. Exp. Neurol. 130; 269-278.

8. Iijima, N., Kataoka, Y., Kakihara, K., Bamba, H., Tamada, Y., Hayashi, S., Matsuda, T., Tanaka, M., Honjyo, H., Hosoya, M., Hinuma, S. and Ibata, Y. (1999) Cytochemical study of prolactinreleasing peptide (PrRP) in the rat brain. Neuroreport 10; 17131716.

9. Iijima, N., Matsumoto, Y., Yano, T., Tanaka, M., Yamamoto, T., Kakihara, K., Kataoka, Y., Tamada, Y., Matsumoto, H., Suzuki, N., Hinuma, S. and Ibata, Y. (2001) A novel function of prolactin-releasing peptide in the control of growth hormone via secretion of somatostatin from the hypothalamus. Endocrinology 142; 3239-3243.

10. Kataoka, Y., Iijima, N., Yano, T., Kakihara, K., Hayashi, S., Hinuma, S., Honjo, H., Hayashi, S., Tanaka, M. and Ibata, Y.
(2001) Gonadal regulation of PrRP mRNA expression in the nucleus tractus solitarius and ventral and lateral reticular nuclei of the rat. Brain Res. Mol. Brain Res. 87; 42-47.

11. Kosik, K. S. and Finch, E. A. (1987) MAP2 and tau segregate into dendritic and axonal domains after the elaboration of morphologically distinct neurites: an immunocytochemical study of cultured rat cerebrum. J. Neurosci. 7; 3142-3153.

12. Maruyama, M., Matsumoto, H., Fujiwara, K., Kitada, C., Hinuma, S., Onda, H., Fujino, M. and Inoue, K. (1999) Immunocytochemical localization of prolactin-releasing peptide in the rat brain. Endocrinology 140; 2326-2333.

13. Maruyama, M., Matsumoto, H., Fujiwara, K., Noguchi, J., Kitada, C., Hinuma, S., Onda, H., Nishimura, O., Fujino, M., Higuchi, T. and Inoue, K. (1999) Central administration of prolactin-releasing peptide stimulates oxytocin release in rats. Neurosci. Lett. 276; 193-196.

14. Maruyama, M., Matsumoto, H., Fujiwara, K., Noguchi, J., Kitada, C., Fujino, M. and Inoue, K. (2001) Prolactin-releasing peptide as a novel stress mediator in the central nervous system. Endocrinology 142; 2032-2038.

15. Matsuda, T., Tamada, Y., Hayashi, S., Iijima, N., Tanaka, M., Yamada, H., Utsunomiya, K., Kudo, M., Kurokawa, K., Kataoka, K., Yanaihara, N., Nagasawa, S. and Ibata, Y. (2001) Secretion of Bax-like protein into systemic circulation from the posterior pituitary in the rat hypothalamo-posterior pituitary system. Acta Histochem. Cytochem. 34; 119-128.

16. Matsumoto, H., Murakami, Y., Horikoshi, Y., Noguchi, J., Habata, Y., Kitada, C., Hinuma, S., Onda, H. and Fujino, M. (1999) Distribution and characterization of immunoreactive prolactin-releasing peptide (PrRP) in rat tissue and plasma. Biochem. Biophys. Res. Commun. 257; 264-268.

17. Matsumoto, H., Maruyama, M., Noguchi, J., Horikoshi, Y., Fujiwara, K., Kitada, C., Hinuma, S., Onda, H., Nishimura, O., Inoue, K. and Fujino, M. (2000) Stimulation of corticotropinreleasing hormone-mediated adrenocorticotropin secretion by central administration of prolactin-releasing peptide in rats. Neurosci. Lett. 285; 234-238.

18. Minami, S., Nakata, T., Tokita, R., Onodera, H. and Imaki, J. (1999) Cellular localization of prolactin-releasing peptide messenger RNA in the rat brain. Neurosci. Lett. 266; 73-75.

19. Morales, T., Hinuma, S. and Sawchenko, P. E. (2000) Prolactinreleasing peptide is expressed in afferents to the endocrine hypothalamus, but not in neurosecretory neurones. J. Neuroendocrinol. $12 ; 131-140$.

20. Nagatsu, I., Kondo, Y., Inagaki, S., Karasawa, N., Kato, T. and Nagatsu, S. (1977) Immunofluorescent studies on tyrosine hydroxylase: application for its axoplasmic transport. Acta Histochem. Cytochem. 10; 494-499.

21. Paxinos, G. and Watson, C. (1998) The Rat Brain Sterotaxic Coordinates, 4th ed., Academic Press.

22. Peng, I., Binder, L. I. and Black, M. M. (1986) Biochemical and immunological analyses of cytoskeletal domains of neurons. J. Cell Biol. 102; 252-262.

23. Roland, B. L., Sutton, S. W., Wilson, S. J., Luo, L., Pyati, J., Huvar, R., Erlander, M. G. and Lovenberg, T. W. (1999) Anatomical distribution of prolactin-releasing peptide and its receptor suggests additional functions in the central nervous system and periphery. Endocrinology 140; 5736-5745.

24. Samson, W. K., Resch, Z. T. and Murphy, T. C. (2000) A novel action of the newly described prolactin-releasing peptides: cardiovascular regulation. Brain Res. 858; 19-25.

25. Tanaka, M., Matsumoto, Y., Murakami, T., Hisa, Y. and Ibata, Y. (1996) The origins of catecholaminergic innervation in the rostral ventromedial medulla oblongata of the rat. Neurosci. Lett. 207; $53-56$.

26. Tanaka, M., Ikeda, T., Hayashi, S., Iijima, N., Amaya, F., Hisa, Y. and Ibata, Y. (1997) Nitrergic neurons in the medial amygdala 
project to the hypothalamic paraventricular nucleus of the rat Brain Res. 777; 13-21.

27. Yano, T., Iijima, N., Kataoka, Y., Hinuma, S., Tanaka, M. and Ibata, Y. (2001) Developmental expression of prolactin releasing peptide in the rat brain: localization of messenger ribonucleic acid and immunoreactive neurons. Brain Res. Dev. Brain Res. $128 ; 101-111$.

28. Zhang, S. Q., Kimura, M. and Inoue, S. (2000) Effects of prolactin-releasing peptide (PrRP) on sleep regulation in rats. Psychiatry Clin. Neurosci. 54; 262-264. 